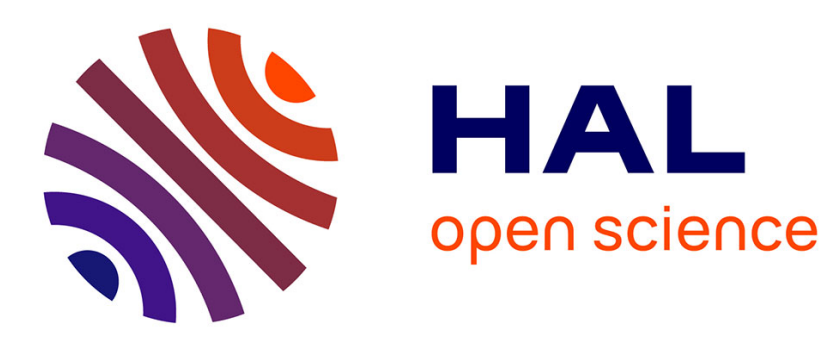

\title{
Phenomenological renormalisation of the self avoiding walk in two dimensions
}

\author{
Bernard Derrida
}

\section{To cite this version:}

Bernard Derrida. Phenomenological renormalisation of the self avoiding walk in two dimensions. Journal of Physics A : Mathematical and General, 1981, 14 (1), pp.L5-L9. 10.1088/0305-4470/14/1/002 . hal-03285947

\section{HAL Id: hal-03285947 \\ https://hal.science/hal-03285947}

Submitted on 21 Jul 2021

HAL is a multi-disciplinary open access archive for the deposit and dissemination of scientific research documents, whether they are published or not. The documents may come from teaching and research institutions in France or abroad, or from public or private research centers.
L'archive ouverte pluridisciplinaire HAL, est destinée au dépôt et à la diffusion de documents scientifiques de niveau recherche, publiés ou non, émanant des établissements d'enseignement et de recherche français ou étrangers, des laboratoires publics ou privés. 


\title{
LETTER TO THE EDITOR
}

\section{Phenomenological renormalisation of the self avoiding walk in two dimensions}

\author{
B Derrida \\ CEN de Saclay, Boite Postale no 2, 91190 Gif-sur-Yvette, France
}

Received 15 October 1980

\begin{abstract}
Phenomenological renormalisation is used to calculate the exponent $\nu$ and the connective constant of the self-avoiding walk problem on a square lattice. A transfer matrix technique is developed for the polymer problem. The results indicate that Flory's value $\nu=0.75$ is true in two dimensions to extremely high accuracy.
\end{abstract}

Trying to calculate the critical properties of the two-dimensional self avoiding walk (SAW) by a real space renormalisation is no longer a very new idea (Hilhorst 1976, 1977, Shapiro 1978, Coniglio and Daoud 1979). However since the phenomenological renormalisation (PR) is becoming more and more useful to study two-dimensional systems (Nightingale 1976, 1979, Sneddon 1978, 1979, Nightingale and Blöte 1980, Ràcz 1980, Derrida and Vannimenus 1980a), I found it interesting to use it in this case. The motivations for the present work were provided by the two following facts. First, the $P R$ requires the calculation of the correlation lengths of strips of finite width. The standard technique to do so for spin systems is the transfer matrix method. It was therefore necessary to define a transfer matrix for the SAW. The procedure is similar to one proposed for percolation (Derrida and Vannimenus 1980a). The interest of the definition given here is that it can be generalised to other polymer problems (solution of polymers, branched polymers, vulcanisation). Secondly, the SAW is simple enough to allow calculations for strips of rather large width. The other purpose of this work was to study the convergence of the PR when the width $n$ of the strip increases. Up to now, the convergence law is not well understood except in the Ising case where the critical temperature and the critical exponent $\nu$ calculated by the PR with strips of width $n$ and $n-1$ and periodic boundary conditions converge respectively like $n^{-3}$ and $n^{-2}$ to their exact values. (Nightingale 1976, Derrida and Vannimenus 1980b).

The PR method introduced by Nightingale $(1976,1979)$ is based on finite-size scaling arguments. I merely recall here the principle of the method without repeating its justifications. Suppose that one wants to study the critical properties of a twodimensional model with coupling constant $x$. Using the transfer matrix one can calculate the correlation length $\xi_{n}(x)$ of a strip of width $n$. The PR consists in writing a renormalisation equation

$$
(1 / n) \xi_{n}(x)=(1 / m) \xi_{m}\left(x^{\prime}\right)
$$

which expresses the changes of the interaction $x$ associated with the change of scale of ratio $n / m$. The critical point and the exponent $\nu$ of the two-dimensional problem can be 
calculated from equations (2) and (3):

$$
\begin{aligned}
& (1 / n) \xi_{n}\left(x_{\mathrm{c}}\right)=(1 / m) \xi_{m}\left(x_{\mathrm{c}}\right) \\
& \frac{1}{\nu}=\frac{\ln \left[\mathrm{d} \xi_{n} /\left.\mathrm{d} x\right|_{x_{\mathrm{c}}}\left(\mathrm{d} \xi_{m} /\left.\mathrm{d} x\right|_{x_{\mathrm{c}}}\right)^{-1}\right]}{\ln (n / m)}-1 .
\end{aligned}
$$

Other exponents can be calculated by similar formulae.

As far as $n$ and $m$ are finite, the method is an approximation which can be improved by choosing $n$ as large as possible and $m=n-1$ (Nightingale 1976, Derrida and Vannimenus 1980b, dos Santos and Sneddon 1980).

In order to use the PR method for the SAW, one has to define the correlation length as a function of a parameter $x$ in the same way as for spin models. This can be done using the famous $q \rightarrow 0$ limit of the classical $q$-component Heisenberg model (de Gennes 1972, des Cloizeaux 1975, Daoud et al 1975)

$$
\lim _{q \rightarrow 0}\left\langle\boldsymbol{S}_{0} \cdot \boldsymbol{S}_{R}\right\rangle=\sum_{p=0}^{\infty} x^{p} \mathcal{N}_{0 R}(p)=G_{0 R}(x) .
$$

Equation (4) relates the correlation function $\left\langle\boldsymbol{S}_{0} \cdot \boldsymbol{S}_{R}\right\rangle$ of Heisenberg spins located on sites 0 and $R$ to the number of self-avoiding walks $\mathcal{N}_{0 R}(p)$ of length $p$ going from site 0 to site $R, x$ is the nearest-neighbour interaction in the Heisenberg model. When the distance $R$ between the two sites becomes large, the correlation function decreases exponentially in the high-temperature phase. In the $q \rightarrow 0$ limit this defines the correlation length $\xi(x)$ for the polymer problem as a function of $x$ which is a chemical potential of monomers

$$
G_{0 R}(x) \sim \exp (R / \xi(x)) .
$$

It is now possible to explain how $\xi(x)$ can be calculated for a strip of any width. Suppose that sites 0 and $R$ belong to two columns $N_{0}$ and $N_{R}$ on the strip. If one cuts the strip at column $N$ between $N_{0}$ and $N_{R}$, the part of the polymer at the left of column $N$ is made of several branches: one site of column $N$ is connected to site 0 of column $N_{0}$ whereas some of the other sites of column $N$ are connected by pairs (figure 1). The writing of the transfer matrix needs two steps.

First, one needs the list of all the possible configurations $\mathscr{C}$ at column $N$. One configuration is defined by the site of column $N$ connected to site 0 and by the pairs of sites connected by the part of the strip at the left of column $N$. Configurations $\mathrm{A}$ and $\mathrm{B}$ of figure 1 are examples of such configurations. One can notice that the different branches which reach column $N$ will be connected together by the right part of the strip to form a single polymer. So, some configurations are eliminated (like configuration C of figure 1) where there are crossings between the different branches of the configuration.

For each allowed configuration $\mathscr{C}$, one can define the function $H_{N}(\mathscr{C})$ by

$$
H_{N}(\mathscr{C})=\sum_{p=0}^{\infty} x^{p} \mathcal{N}_{N}(p, \mathscr{C})
$$

where $\mathcal{N}_{N}(p, \mathscr{C})$ is the number of ways one can put $p$ monomers at the left of column $N$ in order to realise configuration $\mathscr{C}$ at column $N$. The transfer matrix $T$ is defined by the 


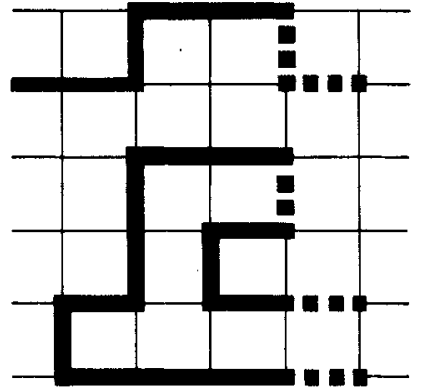

$\leftarrow N_{0}$

$$
N \quad N+1 \quad N_{R} \rightarrow
$$

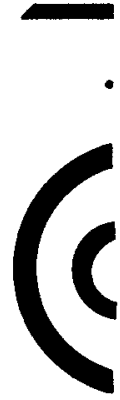

A
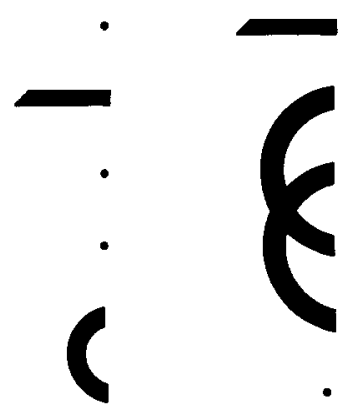

Figure 1. The configurations $A$ and $B$ represent the part of the polymer on the strip at the left of columns $N$ and $N+1$ respectively. The dashed links are the monomers one has to add to configuration $\mathrm{A}$ at column $N$ to give rise to configuration $\mathrm{B}$ at column $N+1$. The number of these monomers is here five. So the matrix element between these configurations $A$ and $\mathrm{B}$ is $x^{5}$. Configuration $\mathrm{C}$ is an example of a forbidden configuration.

set of linear relations which allow the calculation of the $H_{N+1}(\mathscr{C})$ as functions of the $H_{N}(\mathscr{C})$ :

$$
H_{N+1}(\mathscr{C})=\sum_{\mathscr{C}^{\prime}} T\left(\mathscr{C}, \mathscr{C}^{\prime}\right) H_{N}\left(\mathscr{C}^{\prime}\right)
$$

Obviously $T\left(\mathscr{C}, \mathscr{C}^{\prime}\right)=x^{t\left(\mathscr{C}, \mathscr{C}^{\prime}\right)}$ where $t\left(\mathscr{C}, \mathscr{C}^{\prime}\right)$ is the number of monomers one has to add to configuration $\mathscr{C}^{\prime}$ at column $N$ to give rise to configuration $\mathscr{C}$ at column $N+1$. If there is no way to connect two configurations $\mathscr{C}$ and $\mathscr{C}^{\prime}$, the matrix element $T\left(\mathscr{C}, \mathscr{C}^{\prime}\right)$ is zero.

So the size of the transfer matrix is the number of configurations $\mathscr{C}$ and its elements are either zeros or integral powers of $x$. Clearly, when the two columns $N_{0}$ and $N_{R}$ are very far from one another, one has

$$
G_{0 R}(x) \sim[\lambda(x)]^{R}
$$

where $\lambda(x)$ is the largest eigenvalue of the matrix $T$. So the correlation length $\xi(x)$ can be calculated by

$$
\xi(x)=-\frac{1}{\ln (\lambda(x))}
$$

From a practical point of view, the size of the matrix can be reduced using the symmetries of the strip. These reduced sizes $S_{n}$ for strips of width $n$ with periodic boundary conditions and free boundary conditions are given in tables 1 and 2 respectively. The general expression of these sizes is too complicated to be given here. Let me just mention that for large $n, S_{n}$ increases like $3^{n}$.

The self avoiding walk was studied here on a square lattice. The results shown in table 1 were obtained by calculating $\xi_{n}(x)$ for strips with periodic boundary conditions and by using formulae (2) and (3) with $m=n-1$. The values of $\nu$ are in much better agreement with Flory's value $\nu=0.75$ (Domb 1969) than previous real-space renormalisations ( $\nu=0.740$ by Hilhorst 1977 and $\nu=0.70$ by Shapiro 1978). The discrepancy between those previous works and Flory's value is however, recovered here for narrow strips and is probably due to small size effects. 
Table 1. Results of the phenomenological renormalisation for the SAw problem using strips of width $n$ and $n-1$ with periodic boundary conditions. $S_{n}$ is the size of the transfer matrix for a strip of width $n$ once the symmetries have been used. The uncertainty on $x_{\mathrm{c}}$ and $\nu$ calculated for all the choices of $n$ and $m$ is less than $10^{-7}$ for $x_{\mathrm{c}}$ and $10^{-6}$ for $\nu$. The error bars indicated for the extrapolated values come only from the extrapolation procedure.

\begin{tabular}{rcccr}
\hline$n$ & $m$ & \multicolumn{1}{c}{$x_{\mathrm{c}}$} & \multicolumn{1}{c}{$\nu$} & $S_{n}$ \\
\hline 2 & 1 & 0.3478104 & 0.668473 & 1 \\
3 & 2 & 0.3653048 & 0.724477 & 2 \\
4 & 3 & 0.3733995 & 0.739124 & 3 \\
5 & 4 & 0.3766329 & 0.745005 & 7 \\
6 & 5 & 0.3779095 & 0.747680 & 13 \\
7 & 6 & 0.3784477 & 0.748928 & 32 \\
8 & 7 & 0.3786984 & 0.749527 & 70 \\
9 & 8 & 0.3788280 & 0.749826 & 179 \\
10 & 9 & 0.3789013 & 0.749983 & 435 \\
11 & 10 & 0.3789459 & 0.750067 & 1142 \\
12 & 11 & & & 2947 \\
Extrapolation & $0.37905 \pm 0.00003$ & $0.7503 \pm 0.0002$ & \\
Flory's value & & 0.75 & \\
\hline
\end{tabular}

Table 2 contains the results of the PR for strips with free boundary conditions. The results are worse than in the periodic case and the convergence of $\nu$ is not even monotonic. However, for large width the agreement with the periodic case seems to take shape.

The extrapolation of the results of table 1 leads to

$$
\begin{aligned}
& \nu=0.7503 \pm 0.0002 \\
& x_{\mathrm{c}}=0.37905 \pm 0.00003
\end{aligned}
$$

and the convergence law is well described by a power law $\left(n^{-3.5}\right)$ for $\nu$ and $x_{\mathrm{c}}$. The Flory value $\nu=\frac{3}{4}$ and the connective constant $x_{c}=0.37900$ given by McKenzie (1976) are out of the error bars of equations (10). However, one cannot be sure that the asymptotic regime in $n$ has been reached. Non-monotonic convergence might occur for larger sizes

Table 2. As table 1 but with free boundary conditions. Note that the results for $\nu$ do not converge monotonically.

\begin{tabular}{rrllr}
\hline$n$ & $m$ & $x_{\mathrm{c}}$ & $\nu$ & \\
\hline 2 & 1 & 0.4655712 & 0.715312 & 1 \\
3 & 2 & 0.4146801 & 0.745306 & 3 \\
4 & 3 & 0.3982330 & 0.753958 & 6 \\
5 & 4 & 0.3908852 & 0.756904 & 16 \\
6 & 5 & 0.3870023 & 0.757908 & 38 \\
7 & 6 & 0.3847187 & 0.758139 & 100 \\
8 & 7 & 0.3832708 & 0.758037 & 256 \\
9 & 8 & 0.3823000 & 0.757790 & 681 \\
10 & 9 & 0.3816200 & 0.757483 & 1805 \\
11 & 10 & & & 4867 \\
\hline
\end{tabular}


as was the case in table 2. In any case, even if Flory's values were not exact, it would be an extremely good approximation to the true exponent.

This work has shown once more that phenomenological renormalisation is a very powerful tool. Small calculations $(n \leqslant 5)$ lead to satisfactory results whereas longer ones give very accurate numbers. It would be of great interest to be able to predict the convergence law of the results for making very accurate extrapolations.

This work was done partly during my visit to the T J Watson Research Center of IBM. I should like to thank $J$ des Cloizeaux, $S$ Kirkpatrick and $P$ Moussa for numerous discussions as well as $\mathrm{H} \mathrm{J}$ Hilhorst who suggested this calculation to me.

\section{References}

des Cloizeaux J 1975 J. Physique 36281

Coniglio A and Daoud M 1979 J. Phys. A: Math. Gen. 12 L259

Daoud M, Cotton J P, Farnoux B, Jannink G, Sarma G, Benoit H, Duplessix R, Picot C and de Gennes P G 1975 Macromolecules 8804

Derrida B and Vannimenus J 1980a J. Physique Lett. 41 in press

— $1980 \mathrm{~b}$ Colloque sur les méthodes de calcul pour l'étude de phénomènes critiques de Carry le Rouet 1980 (Berlin: Springer)

Domb C 1969 Adv. Chem. Phys, 15229

de Gennes P G 1972 Phys. Lett. 38A 339

Hilhorst H J 1976 Phys. Lett. A38 339 1977 Phys. Rev. B16 1253

McKenzie D S 1976 Phys. Rep. 27C 37

Nightingale M P 1976 Physica 83A 561

1979 Proc. Koninklijke Nederlandse Akademic van Wetenschappen $\mathbf{B 8 2}$ (3) 235

Nightingale M P and Blöte H W J Physica to be published

Ràcz Z 1980 Phys. Rev. B21 4012

dos Santos R R and Sneddon L 1980 Preprint

Shapiro B 1978 J. Phys. C: Solid State Phys. 112829

Sneddon L 1978 J. Phys. C: Solid State Phys. 112823

1979 J. Phys. C: Solid State Phys. 123051 